\title{
Performance evaluation of Black Hole Algorithm, Gravitational Search Algorithm and Particle Swarm Optimization
}

\author{
Mohamad Nizam Aliman ${ }^{1}$, Zuwairie Ibrahim¹, Fardila Naim¹, Sophan Wahyudi Nawawi², Shahdan Sudin² \\ ${ }^{1}$ Engineering Optimization Research Group, Faculty of Electronics \& Electrical Engineering, Universiti Malaysia Pahang, 26300 Kuantan, Pahang, \\ Malaysia. \\ ${ }^{2}$ Faculty of Electrical Engineering, Universiti Teknologi Malaysia, 81310 UTM Johor Bahru, Johor, Malaysia \\ *Corresponding Author: mohamad.aliman@gmail.com
}

Article history :

Received 3 September 2014

Accepted 16 February 2015

GRAPHICAL ABSTRACT

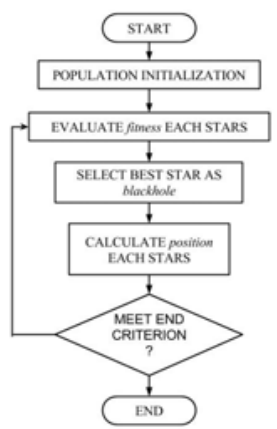

\section{ABSTRACT}

Particle Swarm Optimization (PSO) and Gravitational Search Algorithm are a well-known population-based heuristic optimization techniques. PSO is inspired from a motion flock of birds searching for a food. In PSO, a bird adjusts its position according to its own "experience"' as well as the experience of other birds. Tracking and memorizing the best position encountered build bird's experience which will leads to optimal solution. GSA is based on the Newtonian gravity and motion laws between several masses. In GSA, the heaviest mass presents an optimum solution in the search space. Other agents inside the population are attracted to heaviest mass and will finally converge to produce best solution. Black Hole Algorithm (BH) is one of the optimization technique recently proposed for data clustering problem. BH algorithm is inspired by the natural universe phenomenon called "black hole”. In BH algorithm, the best solution is selected to be the black hole and the rest of candidates which are called stars will be drawn towards the black hole. In this paper, performance of $\mathrm{BH}$ algorithm will be analyzed and reviewed for continuous search space using CEC2014 benchmark dataset against Gravitational Search Algorithm (GSA) and Particle Swarm Optimization (PSO). CEC2014 benchmark dataset contains 4 unimodal, 7 multimodal and 6 hybrid functions. Several common parameters has been chosen to make an equal comparison between these algorithm such as size of population is 30, 1000 iteration, 30 dimension and 30 times of experiment.

Keyword : black hole algorithm, nature inspire metaheuristic

(C) 2015 Penerbit UTM Press. All rights reserved http://dx.doi.org/10.11113/mjfas.v11n1.342

\section{INTRODUCTION}

Optimization is an applied science which explores the best values of the parameters of a problem that may take under specified conditions [1][2]. The design of an optimization problem generally starts with the design of an objective function [3][4][5][6]. The metaheuristic optimization algorithms use two basic strategies while searching for the global optimum; exploration and exploitation [3].

There are numerous metaheuristic optimization algorithms to date. Those algorithms are Ant Colony Algorithm [7], Firefly Algorithm [8], Artificial Bee Colony [4], Cuckoo Search Algorithm [9], Harmony Search Algorithm [10]. However, in this study, swarm intelligence algorithms, which are part of metaheuristic optimization algorithms, are studied. In particular, the performance of particle swarm optimization (PSO), gravitational search algorithm (GSA), and the most recent black hole algorithm $(\mathrm{BH})$ are evaluated based on the latest benchmark functions called CEC2014 benchmark functions [11]. The purpose of this study is to explore the capability of PSO, GSA, and BH algorithms and to obtain a general conclusion regarding which one is the best among others.

The paper is organized as follows: Section 2 present a brief introduction to all algorithms involved; PSO, GSA and BH. Section 3 describe about benchmark functions, common setting and parameter will be used in the experiment. The experimental result and discussion are provided in Section 4. Finally, Section 5 concludes the work.

\section{ALGORITHM}

\subsection{Particle Swarm Optimization (PSO)}

PSO is a stochastic global optimization technique inspired by social behaviour of bird flocking or fish schooling [12]. PSO uses simple mechanism observed from swarm behaviour to guide particles in search for a global optimal solution. In PSO, each particle moving inside search space with a velocity dynamically adjusted according to its own previous best position and its neighbourhood best position. Hence, every particle is representing as a potential optimal solution for the problem. Initially, each particle is randomly placed inside of $d$-dimensional search space. The $i$ th particle is represented as $X_{i}=\left(x_{i}^{1} \ldots x_{i}^{d} \ldots x_{i}^{n}\right)$.

At the specific time " $t$ ", the velocity for $i$ th particle is calculated using below formula:

$$
\begin{aligned}
& v_{i}^{d}(t+1)=\omega(t) v_{i}^{d}(t)+c_{1} \operatorname{rand}_{i 1}\left(\text { pbest }_{i}^{d}-x_{i}^{d}(t)\right)+ \\
& c_{2} \operatorname{rand}_{i 2}\left(\operatorname{gbest}^{d}-x_{i}^{d}(t)\right)
\end{aligned}
$$

Where, pbest $_{i}$ represent best previous position of the $i$ th particle and $g$ best represent best previous position 
among all the particles in the population. Particle position for the next iteration is calculated as follow:

$x_{i}^{d}(t+1)=x_{i}^{d}(t)+v_{i}^{d}(t+1)$

The general principle of PSO is shown in Figure 1.

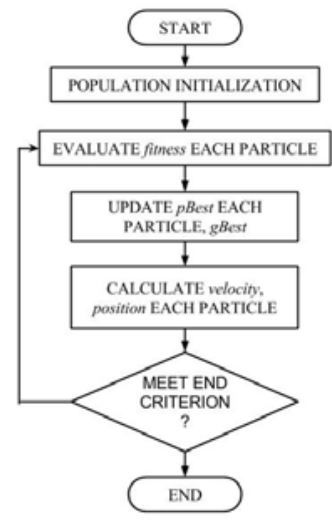

Figure 1. General principle of PSO

\subsection{Gravitational Search Algorithm (GSA)}

GSA has been inspired from physical phenomenon of interaction between objects in the universe. It is defined by Newton as, "Every particle in the universe attract every other particle with a force that is directly proportional to the square of the distance between them". This definition is known as gravitational force and is defined as follow:

$F=\frac{G M_{1} M_{2}}{R^{2}}$

In GSA, agents are considered as objects and their performance are expressed by their masses [3] value which calculated from specific fitness function. The population will be initialized by placing the agent at randomly position inside search space. Assuming gravitational and inertia mass is equal, agents masses are calculated using following equations:

$\operatorname{best}(t)=\max _{j \in\{1, \ldots, N\}} f_{i t}(t)$

$\operatorname{worst}(t)=\min _{j \in\{1, \ldots, N\}}$ fit $_{j}(t)$

$M_{a i}=M_{p i}=M_{i i}$ where $M_{i}, i=1,2,3 \ldots N$

$m_{i}(t)=\frac{\operatorname{fit}_{i}(t)-\operatorname{worst}(t)}{\operatorname{best}(t)-\operatorname{worst}(t)}$

$M_{i}(t)=\frac{m_{i}(t)}{\sum_{j=1}^{N} m_{j}(t)}$

So, at specific time " $t$ ", the gravitational force acting on agent " $i$ ” from agent " $j$ ” can be represent as following:

$F_{i j}^{d}(t)=G(t) \frac{M_{p i}(t) \times M_{a j}(t)}{R_{i j}(t)+\varepsilon}\left(x_{j}^{d}(t)-x_{i}^{d}(t)\right)$

The Euclidian distance between two agents is:

$R_{i j}(t)=\left\|X_{i}(t), X_{j}(t)\right\|_{2}$

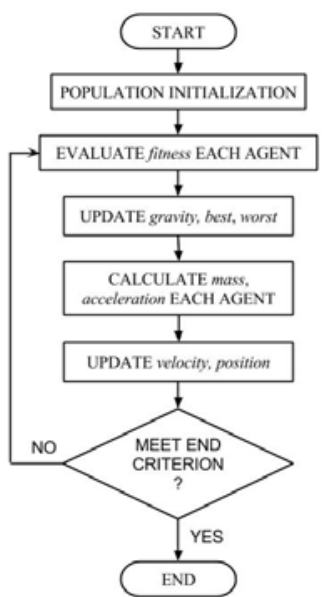

Figure 2. General principle of GSA

The gravitational coefficient $G(t)$ will be reduced with time to control the search accuracy.

$G(t)=G\left(G_{0}, t\right)$

Following formulas has been used to determine the " $i$ "th agent acceleration:

$F_{i}^{d}(t)=\sum_{j=1, j \neq i}^{N} \operatorname{rand}_{i} F_{i j}^{d}(t)$

$a_{i}^{d}(t)=\frac{F_{i}^{d}(t)}{M_{i i}(t)}$

Then, the agent new velocity and position are calculated using these equations:

$v_{i}^{d}(t+1)=\operatorname{rand}_{i} \times v_{i}^{d}(t)+a_{i}^{d}(t)$

$x_{i}^{d}(t+1)=x_{i}^{d}(t)+v_{i}^{d}(t+1)$

The general principle of GSA is shown in Figure 2.

\subsection{Black Hole Algorithm (BH)}

$\mathrm{BH}$ was created from a black hole phenomenon. It was first intended to be use as an alternative for clustering problem. Black hole phenomenon has been named by John Wheeler an American Physicist in 1967. It is a space having a huge gravitational power in which anything crosses the boundary will be swallowed even the light. A black hole in space is what forms when a star of massive size collapses [13].

Imagined an $n$-population of stars with initially placed inside of $d$-dimensional search space as follow:

$X_{i}=\left(x_{i}^{1} \ldots x_{i}^{d} \ldots x_{i}^{n}\right)$

Then, each star will be evaluated using chosen fitness function. Star having the best fitness value will be selected to become a black hole. The new position for all stars will be calculated using this formula:

$x_{i}^{d}(t+1)=x_{i}^{d}(t)+\operatorname{rand} \times\left(x_{B H}^{d}-x_{i}^{d}(t)\right)$ 


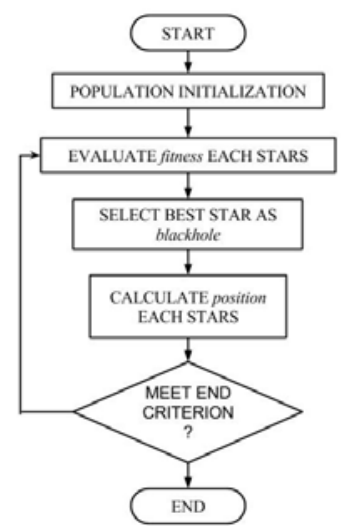

Figure 3. Standard BH flowchart

The new star will be created at random location every time it cross event horizon. Event horizon is a radius boundary around black hole. The value is determined by the following formula:

$R=\frac{\text { fitness }_{B H}}{\sum_{i=1}^{N} \text { fitness }_{i}}$ where $N=$ population size

The general principle of $\mathrm{BH}$ is shown in Figure 3.

\section{EXPERIMENTS, RESULTS AND DISCUSSION}

The parameter setting of PSO, GSA, and $\mathrm{BH}$ are shown in Table 1 . Number of run, number of iteration, population size, and number of dimension are general parameter applied to all algorithms. Inertia weight $(\omega)$, cognitive $\left(c_{1}\right)$ and social $\left(c_{2}\right)$ parameters are exclusive to PSO. On the other hand, initial gravitation constant $\left(G_{0}\right)$ and alpha $(\alpha)$, are exclusive to GSA.

The experiments were based on the recently published benchmark functions called CEC2014 benchmark functions [11]. The formulation of the CEC2014 benchmark functions are listed in Table 6 and Table 7. The benchmark functions are divided into unimodal, multimodal, and hybrid functions with optimal value are also included.

Table 1 Parameter setting

\begin{tabular}{|c|c|c|}
\hline & Parameter & Value \\
\hline \multirow{4}{*}{ تٍِّ } & Number of run per experiment & 30 \\
\hline & Number of iteration per run & 1000 \\
\hline & Population size & 30 \\
\hline & Number of dimension & 30 \\
\hline \multirow{2}{*}{ @ } & Inertia Weight, $\omega$ & 0.9 to 0.4 \\
\hline & Coefficient Factor, $c_{1}, c_{2}$ & 2,2 \\
\hline \multirow{2}{*}{ 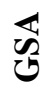 } & Initial Gravity, $G_{0}$ & 100 \\
\hline & Alpha, $\alpha$ & -20 \\
\hline
\end{tabular}

The average value, standard deviation value, minimum value, and maximum value from experimental result were recorded and tabulated in Table 2, Table 3, Table 4 and
Table 5. The average value is being used as comparison between algorithms and value written in bold indicates the best result among them.

Table 2 Unimodal function result

\begin{tabular}{|c|c|c|c|c|}
\hline Function & Measure & BH & GSA & PSO \\
\hline \multirow{4}{*}{ F1 } & AVERAGE & 88570364 & $\mathbf{7 4 8 3 8 8 5 4}$ & 96722332 \\
\cline { 2 - 5 } & STDDEV & 31842233 & 23787602 & 71277779 \\
\cline { 2 - 5 } & MIN & 25102312 & 38704969 & 25102312 \\
\cline { 2 - 5 } & MAX & $1.51 \mathrm{E}+08$ & $1.24 \mathrm{E}+08$ & $4.06 \mathrm{E}+08$ \\
\hline \multirow{4}{*}{ F2 } & AVERAGE & $1.36 \mathrm{E}+09$ & $3.29 \mathrm{E}+08$ & $\mathbf{5 7 7 2 9 9 2 2}$ \\
\cline { 2 - 5 } & STDDEV & $1.89 \mathrm{E}+09$ & $4.34 \mathrm{E}+08$ & 96226073 \\
\cline { 2 - 5 } & MIN & 226617.4 & 226617.4 & 1005150 \\
\cline { 2 - 5 } & MAX & $8.00 \mathrm{E}+09$ & $2.13 \mathrm{E}+09$ & $4.08 \mathrm{E}+08$ \\
\hline \multirow{4}{*}{ F3 } & AVERAGE & 43136.29 & 76896.34 & 14178.49 \\
\cline { 2 - 5 } & STDDEV & 27926.54 & 5996.396 & 14777.49 \\
\cline { 2 - 5 } & MIN & 1150.474 & 64485.01 & 1150.474 \\
\cline { 2 - 5 } & MAX & 48712.46 & 87213.57 & 59125.39 \\
\hline
\end{tabular}

Table 3 Multimodal function result.

\begin{tabular}{|c|c|c|c|c|}
\hline Function & Measure & $\mathrm{BH}$ & GSA & PSO \\
\hline \multirow{4}{*}{ F4 } & AVERAGE & 1046.042 & 976.161 & 1031.017 \\
\hline & STDDEV & 277.3205 & 289.4972 & 262.6738 \\
\hline & MIN & 578.9538 & 732.3658 & 578.9538 \\
\hline & MAX & 2136.106 & 2177.511 & 1513.875 \\
\hline \multirow{4}{*}{ F5 } & AVERAGE & 520.306 & 519.999 & 520.9736 \\
\hline & STDDEV & 0.453085 & 0.000556 & 0.101111 \\
\hline & MIN & 519.9975 & 519.9975 & 520.6635 \\
\hline & MAX & 520.0637 & 519.9997 & 521.0941 \\
\hline \multirow{4}{*}{ F6 } & AVERAGE & 624.9259 & 624.7608 & 617.3393 \\
\hline & STDDEV & 6.816158 & 2.449066 & 4.123768 \\
\hline & MIN & 610.2717 & 621.1697 & 610.2717 \\
\hline & MAX & 638.7539 & 629.5551 & 626.4682 \\
\hline \multirow{4}{*}{ F7 } & AVERAGE & 720.3244 & 706.2988 & 710.0631 \\
\hline & STDDEV & 19.22478 & 3.606268 & 11.96609 \\
\hline & MIN & 701.0355 & 701.0355 & 701.2862 \\
\hline & MAX & 767.6485 & 714.0935 & 747.8384 \\
\hline
\end{tabular}

For unimodal functions, GSA is better than PSO and $\mathrm{BH}$ for $\mathrm{F} 1$ function. However, PSO is better than GSA and $\mathrm{BH}$ for F2 and F3 functions while $\mathrm{BH}$ was not able to outperform PSO and GSA in all cases. The examples of boxplot and convergence curves for F1, F2, and F3 functions are shown in Figure 4, Figure 5, and Figure 6 respectively.

Similar to unimodal functions, multimodal functions also shows that $\mathrm{BH}$ was not able to outperform PSO and GSA in all cases. Further comparisons of PSO and GSA show that GSA outperformed PSO in 7 cases (F4, F5, F6, F7, F8, F9, and F10). On the other hand, PSO outperformed GSA in 6 cases (F11, F12, F13, F14, F15, and F16). The examples of boxplot and convergence curves for F4 to F16 are shown in Figure 7 to Figure 18 respectively.

As for hybrid cases, the result show that PSO is superior to GSA and $\mathrm{BH}$ in all cases. The examples of boxplot and convergence curves for F17, F18, F19, F20, F21, and F22 are shown in Figure 19 to Figure 24, respectively. 
Table 4 Multimodal function result.

\begin{tabular}{|c|c|c|c|c|}
\hline Function & Measure & $\mathrm{BH}$ & GSA & PSO \\
\hline \multirow{4}{*}{ F8 } & AVERAGE & 913.3048 & 947.4192 & 854.8536 \\
\hline & STDDEV & 42.41577 & 9.564984 & 13.18151 \\
\hline & MIN & 834.6769 & 929.3442 & 834.6769 \\
\hline & MAX & 971.3446 & 963.1726 & 882.0581 \\
\hline \multirow{4}{*}{ F9 } & AVERAGE & 1110.987 & 1077.898 & 1013.347 \\
\hline & STDDEV & 97.44744 & 20.3977 & 39.0376 \\
\hline & MIN & 959.6918 & 1043.274 & 959.6918 \\
\hline & MAX & 1268.172 & 1127.844 & 1095.535 \\
\hline \multirow{4}{*}{ F10 } & AVERAGE & 3481.428 & 4617.347 & 2027.867 \\
\hline & STDDEV & 1132.673 & 425.2253 & 371.118 \\
\hline & MIN & 1262.792 & 3669.495 & 1262.792 \\
\hline & MAX & 4331.487 & 5230.704 & 2759.996 \\
\hline \multirow{4}{*}{ F11 } & AVERAGE & 5870.741 & 5350.104 & 7166.539 \\
\hline & STDDEV & 1196.285 & 352.6554 & 1081.404 \\
\hline & MIN & 3860.466 & 4685.935 & 3984.792 \\
\hline & MAX & 6526.11 & 6189.264 & 8707.647 \\
\hline \multirow{4}{*}{ F12 } & AVERAGE & 1201.036 & 1200.016 & 1202.343 \\
\hline & STDDEV & 1.03915 & 0.008515 & 0.536511 \\
\hline & MIN & 1200.005 & 1200.005 & 1201.291 \\
\hline & MAX & 1201.644 & 1200.044 & 1203.383 \\
\hline \multirow{4}{*}{ F13 } & AVERAGE & 1300.821 & 1300.515 & 1300.987 \\
\hline & STDDEV & 0.633971 & 0.373776 & 0.708315 \\
\hline & MIN & 1300.203 & 1300.226 & 1300.203 \\
\hline & MAX & 1302.185 & 1302.164 & 1302.704 \\
\hline \multirow{4}{*}{ F14 } & AVERAGE & 1412.875 & 1404.689 & 1419.276 \\
\hline & STDDEV & 13.07767 & 9.784895 & 16.72074 \\
\hline & MIN & 1400.201 & 1400.201 & 1400.207 \\
\hline & MAX & 1439.243 & 1435.619 & 1453.36 \\
\hline \multirow{4}{*}{ F15 } & AVERAGE & 1574.77 & 1554.118 & 1520.718 \\
\hline & STDDEV & 62.15823 & 22.23178 & 5.288553 \\
\hline & MIN & 1508.884 & 1522.382 & 1508.884 \\
\hline & MAX & 1811.993 & 1597.114 & 1534.195 \\
\hline \multirow{4}{*}{ F16 } & AVERAGE & 1612.951 & 1613.61 & 1612.529 \\
\hline & STDDEV & 0.635518 & 0.225436 & 0.45425 \\
\hline & MIN & 1611.25 & 1613.185 & 1611.37 \\
\hline & MAX & 1613.535 & 1613.975 & 1613.24 \\
\hline
\end{tabular}

Table 5 Hybrid function result.

\begin{tabular}{|c|c|c|c|c|}
\hline Function & Measure & BH & GSA & PSO \\
\hline \multirow{4}{*}{ F17 } & AVERAGE & 30784257 & 55283638 & 19729686 \\
\cline { 2 - 5 } & STDDEV & 44065650 & 67306551 & 20705286 \\
\cline { 2 - 5 } & MIN & 1335720 & 3167296 & 1335720 \\
\cline { 2 - 5 } & MAX & 25736003 & $3.28 \mathrm{E}+08$ & $1.04 \mathrm{E}+08$ \\
\hline \multirow{4}{*}{ F18 } & AVERAGE & $6.38 \mathrm{E}+08$ & $1.76 \mathrm{E}+09$ & 32246294 \\
\cline { 2 - 5 } & STDDEV & $1.19 \mathrm{E}+09$ & $1.51 \mathrm{E}+09$ & $1.45 \mathrm{E}+08$ \\
\cline { 2 - 5 } & MIN & 2075.141 & 2519.887 & 2075.141 \\
\cline { 2 - 5 } & MAX & $1.43 \mathrm{E}+08$ & $5.06 \mathrm{E}+09$ & $7.88 \mathrm{E}+08$ \\
\hline \multirow{4}{*}{ F19 } & AVERAGE & 2045.871 & 2105.174 & $\mathbf{2 0 2 1 . 9 7 3}$ \\
\cline { 2 - 5 } & STDDEV & 63.20811 & 42.23975 & 64.39642 \\
\cline { 2 - 5 } & MIN & 1922.153 & 2001.32 & 1922.153 \\
\cline { 2 - 5 } & MAX & 2042.365 & 2222.969 & 2184.441 \\
\hline \multirow{5}{*}{ F20 } & AVERAGE & 57585.45 & 91579.14 & 41567.73 \\
\cline { 2 - 5 } & STDDEV & 30545.26 & 24091.65 & 16684.78 \\
\cline { 2 - 5 } & MIN & 18416.79 & 52543.42 & 20306.05 \\
\cline { 2 - 5 } & MAX & 65022.72 & 153681.4 & 96529.06 \\
\hline \multirow{4}{*}{ F21 } & AVERAGE & 3824788 & 4337785 & 3445418 \\
\cline { 2 - 5 } & STDDEV & 2321736 & 1783216 & 3219689 \\
\cline { 2 - 5 } & MIN & 612435.2 & 1888554 & 612435.2 \\
\cline { 2 - 5 } & MAX & 7217596 & 8681296 & 17397691 \\
\hline \multirow{5}{*}{ F22 } & AVERAGE & 4341.088 & 4544.53 & 4169.702 \\
\cline { 2 - 5 } & STDDEV & 537.9198 & 462.2675 & 629.633 \\
\cline { 2 - 5 } & MIN & 2967.647 & 3832.037 & 2967.647 \\
\cline { 2 - 5 } & MAX & 5000.246 & 6206.148 & 5370.399 \\
\hline \multirow{4}{*}{} & & & & \\
\hline & & &
\end{tabular}

\section{CONCLUSIONS}

This study considers three different swarm intelligence algorithms, namely PSO, GSA, and BH. The purpose of this study is to evaluate the superiority of these algorithms when finding the optimal solution based on CEC2014 benchmark functions.

By observing the results produced based on the unimodal, multimodal, and hybrid functions of CEC2014, it can be concluded that briefly, both PSO and GSA perform well in solving unimodal and multimodal optimization problems. However, for the case of hybrid optimization problem, PSO is superior to GSA and $\mathrm{BH}$ for all cases. The next step of this research are to reexecute similar experiments for high-dimensional optimization problem and to perform a detailed statistical analysis in order to obtain a more concrete conclusion as well as to further understand the behaviour of the PSO, GSA, and BH algorithms.

\section{ACKNOWLEDGMENT}

This work is financially supported by the RAGS (RDU121403) awarded by the Ministry of Higher Education (MOHE) to Universiti Malaysia Pahang (UMP). The first author is thankful to Universiti Malaysia Pahang (UMP) for granting him an opportunity to further his study in postgraduate program in UMP.

\section{References}

[1] D. Corne, M. Dorigo, and F. Glover, New Ideas in Optimization. McGraw-Hill, 1999.

[2] R. Horst, P. M. Pardalos, and N. V. Thoai, Introduction to global optimization, 2nd ed. Dordrecht, The Netherland: Kluwer Academic Publishers, 2000.

[3] E. Rashedi, H. Nezamabadi-pour, and S. Saryazdi, "GSA: A Gravitational Search Algorithm,” Inf. Sci. (Ny)., vol. 179, no. 13, pp. 2232-2248, 2009.

[4] D. Karaboga and B. Akay, “A comparative study of Artificial Bee Colony algorithm,” Appl. Math. Comput., vol. 214, no. 1, pp. 108-132, 2009.

[5] Y. del Valle, G. K. Venayagamoorthy, S. Mohagheghi, J. C. Hernandez, and R. G. Harley, "Particle Swarm Optimization: Basic Concepts, Variants and Applications in Power Systems," Evol. Comput. IEEE Trans., vol. 12, no. 2, pp. 171-195, 2008.

[6] R. Storn and K. Price, "Differential evolution - A simple and efficient heuristic for global optimization over continuous spaces,” J. Glob. Optim., vol. 11, no. 4, pp. 341-359, 1997.

[7] M. Dorigo, M. Birattari, and T. St, “Ant Colony Optimization,” IEEE Computational Intelligence Magazine, no. November, pp. 28-39, 2006.

[8] X. Yang, "Firefly Algorithms for Multimodal Optimization," in Stochastic Algorithms: Foundations and Applications, 2009, pp. 169-178.

[9] R. Rajabioun, “Cuckoo Optimization Algorithm,” Appl. Soft Comput., vol. 11, no. 8, pp. 5508-5518, Dec. 2011.

[10] G. V. Loganathan, "A New Heuristic Optimization Algorithm: Harmony Search,” Simulation, vol. 76, no. 2, pp. 60-68, Feb. 2001.

[11] J. J. Liang, B. Y. Qu, and P. N. Suganthan, Problem Definitions and Evaluation Criteria for the CEC 2014 Special Session and Competition on Single Objective Real-Parameter Numerical Optimization, no. December 2013. 2013.

[12] J. Kennedy and R. Eberhart, "Particle Swarm Optimization," IEEE Int. Conf. Neuron Networks, pp. 1942-1948, 1995.

[13] A. Hatamlou, "Black hole: A new heuristic optimization approach for data clustering," Inf. Sci. (Ny)., vol. 222, pp. 175-184, Feb. 2013. 
Table 6 CEC2014 basic function.

\begin{tabular}{|c|c|}
\hline Function & Equation \\
\hline High Conditioned Elliptic & $f_{1}(\boldsymbol{x})=\sum_{i=1}^{D}\left(10^{6}\right)^{\frac{i-1}{D-1}} \boldsymbol{x}_{i}^{2}$ \\
\hline Bent Cigar & $f_{2}(\boldsymbol{x})=x_{1}^{2}+10^{6} \sum_{i=2}^{D} x_{i}^{2}$ \\
\hline Discuss & $f_{3}(\boldsymbol{x})=10^{6} x_{1}^{2}+\sum_{i=2}^{D} x_{i}^{2}$ \\
\hline Rosenbrock & $f_{4}(\boldsymbol{x})=\sum_{i=1}^{D-1}\left(100\left(x_{i}^{2}-x_{i+1}\right)^{2}+\left(x_{i}-1\right)^{2}\right)$ \\
\hline Ackley & $f_{5}(\boldsymbol{x})=-20 \exp \left(-0.2 \sqrt{\frac{1}{D} \sum_{i=1}^{D} x_{i}^{2}}\right)-\exp \left(\frac{1}{D} \sum_{i=1}^{D} \cos \left(2 \pi x_{i}\right)\right)+20+e$ \\
\hline Weiestrass & $f_{6}(\boldsymbol{x})=\sum_{i=1}^{D}\left(\sum_{k=0}^{k \max }\left[a^{k} \cos \left(2 \pi b^{k}\left(x_{i}+0.5\right)\right)\right]\right)-D \sum_{k=0}^{k \max }\left[a^{k} \cos \left(2 \pi b^{k} \cdot 0.5\right)\right]$ \\
\hline Griewank & $f_{7}(\boldsymbol{x})=\sum_{i=1}^{D} \frac{x_{i}^{2}}{4000}-\prod_{i=1}^{D} \cos \left(\frac{x_{i}}{\sqrt{i}}\right)+1$ \\
\hline Rastrigin & $f_{8}(\boldsymbol{x})=\sum_{i=1}^{D}\left(x_{i}^{2}-10 \cos \left(2 \pi x_{i}\right)+10\right)$ \\
\hline Schwefel & $\begin{array}{l}f_{9}(\boldsymbol{x})=418.9829 \times D-\sum_{i=1}^{D} g\left(z_{i}\right), \\
g\left(z_{i}\right)= \begin{cases}z_{i} \sin \left(\left|z_{i}\right|^{1 / 2}\right) & \text { if }\left|z_{i}\right| \leq 500 \\
\left(500-\bmod \left(z_{i}, 500\right)\right) \sin \left(\sqrt{\left|500-\bmod \left(z_{i}, 500\right)\right|}\right)-\frac{\left(z_{i}-500\right)^{2}}{10000 D} & \text { if } z_{i}>500 \\
\left(\bmod \left(\left|z_{i}\right|, 500\right)-500\right) \sin \left(\sqrt{\left|\bmod \left(\left|z_{i}\right|, 500\right)-500\right|}\right)-\frac{\left(z_{i}+500\right)^{2}}{10000 D} & \text { if } z_{i}<-500\end{cases} \end{array}$ \\
\hline Katsuura & $f_{10}(\boldsymbol{x})=\frac{10}{D^{2}} \prod_{i=1}^{D}\left(1+i \sum_{j=1}^{32} \frac{\left|2^{j} x_{i}-\operatorname{round}\left(2^{j} x_{i}\right)\right|}{2^{j}}\right)^{\frac{10}{D^{12}}}-\frac{10}{D^{2}}$ \\
\hline HappyCat & $f_{11}(\boldsymbol{x})=\left|\sum_{i=1}^{D} x_{i}^{2}-D\right|^{1 / 4}+\left(0.5 \sum_{i=1}^{D} x_{i}^{2}+\sum_{i=1}^{D} x_{i}\right) / D+0.5$ \\
\hline HGBat & $f_{12}(\boldsymbol{x})=\left|\left(\sum_{i=1}^{D} x_{i}^{2}\right)^{2}-\left(\sum_{i=1}^{D} x_{i}\right)^{2}\right|^{1 / 2}+\left(0.5 \sum_{i=1}^{D} x_{i}^{2}+\sum_{i=1}^{D} x_{i}\right) / D+0.5$ \\
\hline $\begin{array}{l}\text { Expanded Griewank plus } \\
\text { Ronsenbrock }\end{array}$ & $f_{13}(\boldsymbol{x})=f_{7}\left(f_{4}\left(x_{1}, x_{2}\right)\right)+f_{7}\left(f_{4}\left(x_{2}, x_{3}\right)\right)+\ldots+f_{7}\left(f_{4}\left(x_{D-1}, x_{D}\right)\right)+f_{7}\left(f_{4}\left(x_{D}, x_{1}\right)\right)$ \\
\hline Expanded Scaffer’s F6 & $\begin{array}{l}f_{14}(\boldsymbol{x})=g\left(x_{1}, x_{2}\right)+g\left(x_{2}, x_{3}\right)+\ldots+g\left(x_{D-1}, x_{D}\right)+g\left(x_{D}, x_{1}\right) \\
g(x, y)=0.5+\frac{\left(\sin ^{2}\left(\sqrt{x^{2}+y^{2}}\right)-0.5\right)}{\left(1+0.001\left(x^{2}+y^{2}\right)\right)^{2}}\end{array}$ \\
\hline Hybrid & 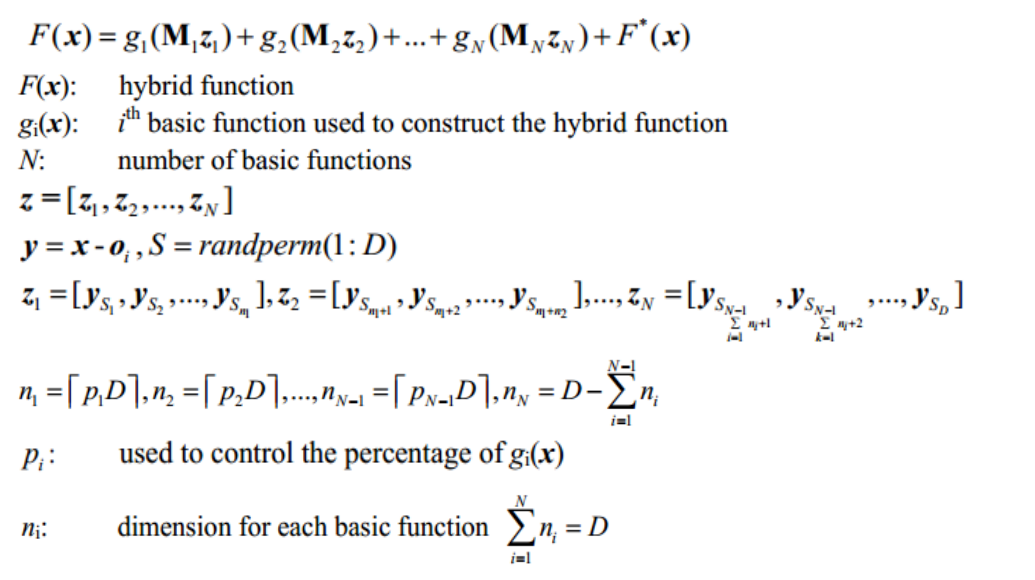 \\
\hline
\end{tabular}


Table 7 CEC2014 benchmarking test function.

\begin{tabular}{|c|c|c|c|}
\hline Type & Function & Equation & Optimal \\
\hline \multirow{3}{*}{ 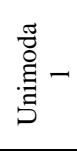 } & Rotated High Conditioned Elliptic & $F_{1}(\boldsymbol{x})=f_{1}\left(\mathbf{M}\left(\boldsymbol{x}-\boldsymbol{o}_{1}\right)\right)+F_{1} *$ & 100 \\
\hline & Rotated Bent Cigar & $F_{2}(\boldsymbol{x})=f_{2}\left(\mathbf{M}\left(\boldsymbol{x}-\boldsymbol{o}_{2}\right)\right)+F_{2} *$ & 200 \\
\hline & Rotated Discuss & $F_{3}(\boldsymbol{x})=f_{3}\left(\mathbf{M}\left(\boldsymbol{x}-\boldsymbol{o}_{3}\right)\right)+F_{3} *$ & 300 \\
\hline \multirow{13}{*}{ 쥴 } & Shifted and rotated Rosenbrock & $F_{4}(\boldsymbol{x})=f_{4}\left(\mathbf{M}\left(\frac{2.048\left(x-o_{4}\right)}{100}\right)+1\right)+F_{4} *$ & 400 \\
\hline & Shifted and rotated Ackley & $F_{5}(\boldsymbol{x})=f_{5}\left(\mathbf{M}\left(\boldsymbol{x}-\boldsymbol{o}_{5}\right)\right)+F_{8} *$ & 500 \\
\hline & Shifted and rotated Weiestrass & $F_{6}(\boldsymbol{x})=f_{6}\left(\mathbf{M}\left(\frac{0.5\left(\boldsymbol{x}-\boldsymbol{o}_{6}\right)}{100}\right)\right)+F_{6} *$ & 600 \\
\hline & Shifted and rotated Griewank & $F_{7}(\boldsymbol{x})=f_{7}\left(\mathbf{M}\left(\frac{600\left(\boldsymbol{x}-\boldsymbol{o}_{7}\right)}{100}\right)\right)+F_{7} *$ & 700 \\
\hline & Shifted Rastrigin & $F_{8}(\boldsymbol{x})=f_{8}\left(\frac{5.12\left(\boldsymbol{x}-\boldsymbol{o}_{8}\right)}{100}\right)+F_{7}^{*}$ & 800 \\
\hline & Shifted and rotated Rastrigin & $F_{9}(\boldsymbol{x})=f_{8}\left(\mathbf{M}\left(\frac{5.12\left(\boldsymbol{x}-\boldsymbol{o}_{9}\right)}{100}\right)\right)+F_{9} *$ & 900 \\
\hline & Shifted Schwefel & $F_{10}(x)=f_{9}\left(\frac{1000\left(x-o_{10}\right)}{100}\right)+F_{10} *$ & 1000 \\
\hline & Shifted and rotated Schwefel & $F_{11}(\boldsymbol{x})=f_{9}\left(\mathbf{M}\left(\frac{1000\left(\boldsymbol{x}-\boldsymbol{o}_{11}\right)}{100}\right)\right)+F_{11} *$ & 1100 \\
\hline & Shifted and rotated Katsuura & $F_{12}(\boldsymbol{x})=f_{10}\left(\mathbf{M}\left(\frac{5\left(\boldsymbol{x}-\boldsymbol{o}_{12}\right)}{100}\right)\right)+F_{12} *$ & 1200 \\
\hline & Shifted and rotated Happycat & $F_{13}(\boldsymbol{x})=f_{11}\left(\mathbf{M}\left(\frac{5\left(\boldsymbol{x}-\boldsymbol{o}_{13}\right)}{100}\right)\right)+F_{13} *$ & 1300 \\
\hline & Shifted and rotated HgBat & $F_{14}(\boldsymbol{x})=f_{12}\left(\mathbf{M}\left(\frac{5\left(\boldsymbol{x}-\boldsymbol{o}_{14}\right)}{100}\right)\right)+F_{14} *$ & 1400 \\
\hline & $\begin{array}{c}\text { Shifted and rotated Expanded Griewank- } \\
\text { Rosenbrock }\end{array}$ & $F_{15}(\boldsymbol{x})=f_{13}\left(\mathbf{M}\left(\frac{5\left(\boldsymbol{x}-\boldsymbol{o}_{15}\right)}{100}\right)+1\right)+F_{15} *$ & 1500 \\
\hline & Shifted and rotated Expanded Scaffer F6 & $F_{16}(\boldsymbol{x})=f_{14}\left(\mathbf{M}\left(\boldsymbol{x}-\boldsymbol{o}_{16}\right)+1\right)+F_{16} *$ & 1600 \\
\hline \multirow{6}{*}{ 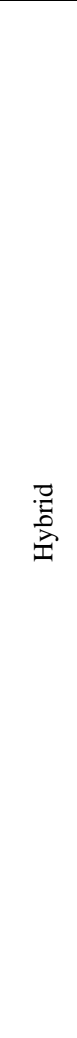 } & Hybrid $1(\mathrm{~N}=3)$ & $\begin{array}{l}p=[0.3,0.3,0.4] \\
g_{1}: \text { Modified Schwefel's Function } f_{9} \\
g_{2}: \text { Rastrigin's Function } f_{8} \\
g_{3}: \text { High Conditioned Elliptic Function } f_{1}\end{array}$ & 1700 \\
\hline & Hybrid $2(\mathrm{~N}=3)$ & $\begin{array}{l}p=[0.3,0.3,0.4] \\
g_{1}: \text { Bent Cigar Function } f_{2} \\
g_{2}: \text { HGBat Function } f_{12} \\
g_{3}: \text { Rastrigin's Function } f_{8}\end{array}$ & 1800 \\
\hline & Hybrid $3(\mathrm{~N}=4)$ & $\begin{array}{l}p=[0.2,0.2,0.3,0.3] \\
g_{1}: \text { Griewank's Function } f_{7} \\
g_{2}: \text { Weierstrass Function } f_{6} \\
g_{3}: \text { Rosenbrock's Function } f_{4} \\
g_{4}: \text { Scaffer's F6 Function: } f_{14}\end{array}$ & 1900 \\
\hline & Hybrid $4(\mathrm{~N}=4)$ & $\begin{array}{l}p=[0.2,0.2,0.3,0.3] \\
g_{1}: \text { HGBat Function } f_{12} \\
g_{2}: \text { Discus Function } f_{3} \\
g_{3}: \text { Expanded Griewank's plus Rosenbrock's Function } f_{13} \\
g_{4}: \text { Rastrigin's Function } f_{8}\end{array}$ & 2000 \\
\hline & Hybrid $5(\mathrm{~N}=5)$ & $\begin{array}{l}p=[0.1,0.2,0.2,0.2,0.3] \\
g_{1}: \text { Scaffer's F6 Function: } f_{14} \\
g_{2}: \text { HGBat Function } f_{12} \\
g_{3}: \text { Rosenbrock's Function } f_{4} \\
g_{4}: \text { Modified Schwefel's Function } f_{9} \\
g_{5}: \text { High Conditioned Elliptic Function } f_{1}\end{array}$ & 2100 \\
\hline & Hybrid $6(\mathrm{~N}=5)$ & $\begin{array}{l}p=[0.1,0.2,0.2,0.2,0.3] \\
g_{1}: \text { Katsuura Function } f_{10} \\
g_{2}: \text { HappyCat Function } f_{11} \\
g_{3}: \text { Expanded Griewank's plus Rosenbrock's Function } f_{13} \\
g_{4}: \text { Modified Schwefel's Function } f_{9} \\
g_{5}: \text { Ackley's Function } f_{5}\end{array}$ & 2200 \\
\hline
\end{tabular}



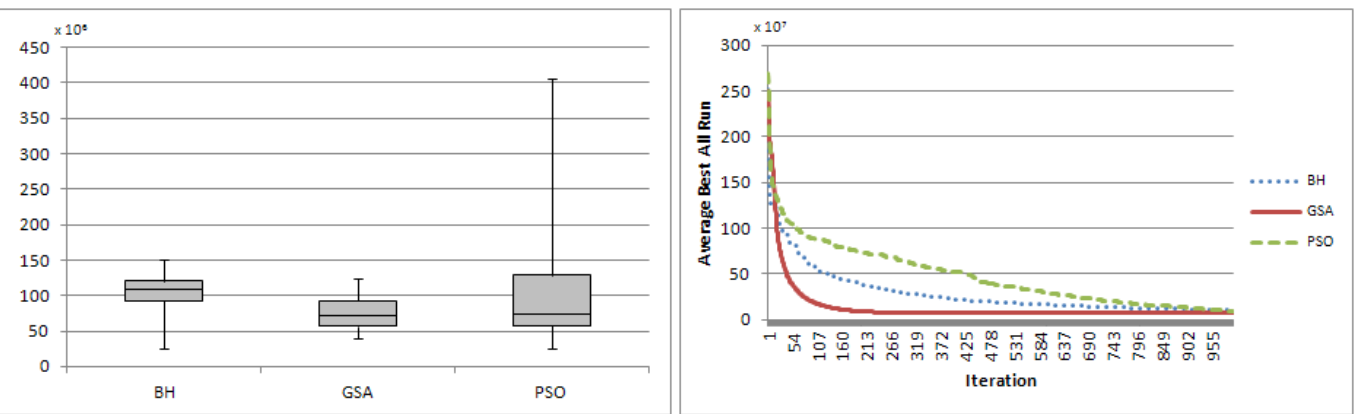

Figure 4 Rotated High Conditioned Elliptic function
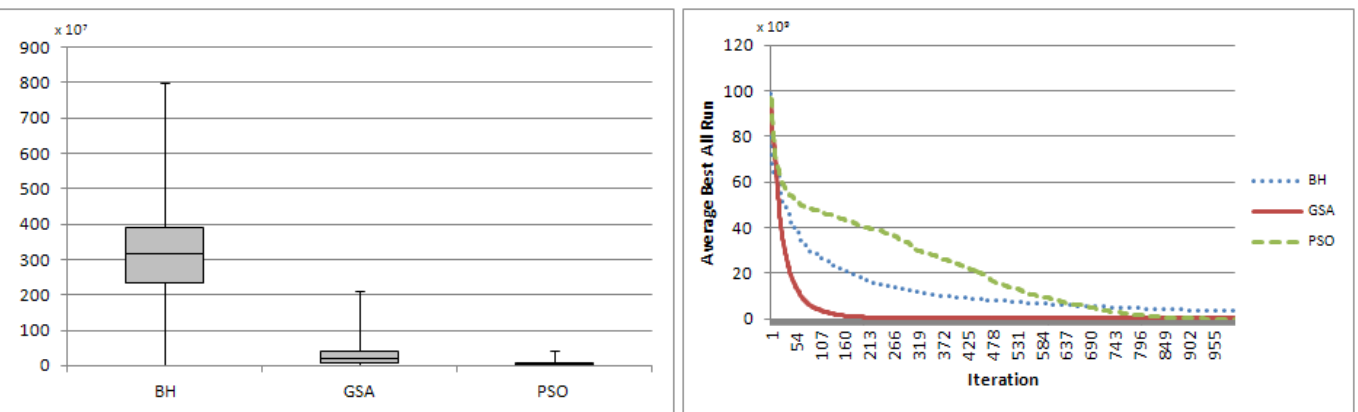

Figure 5 Rotated Bent Cigar function
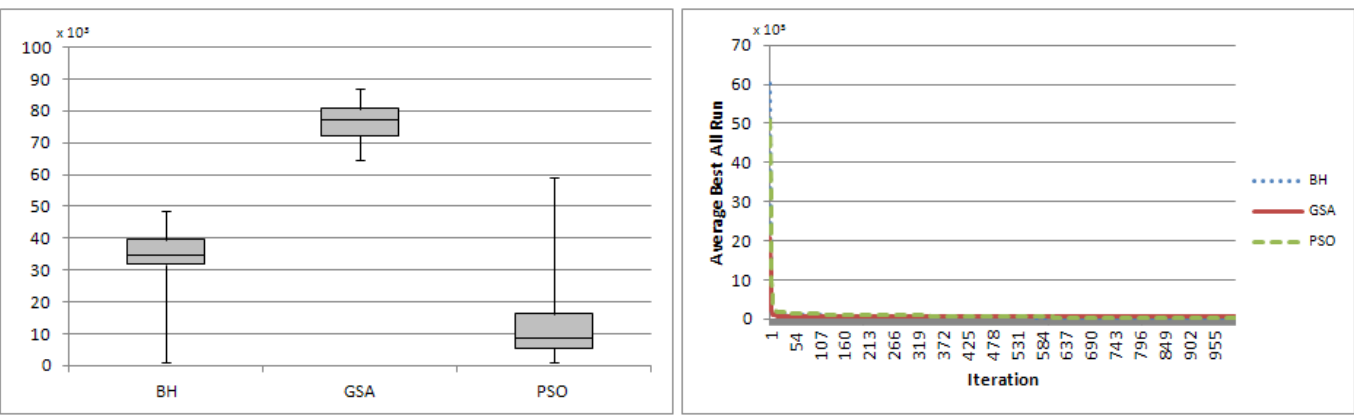

Figure 6: Rotated Discuss function
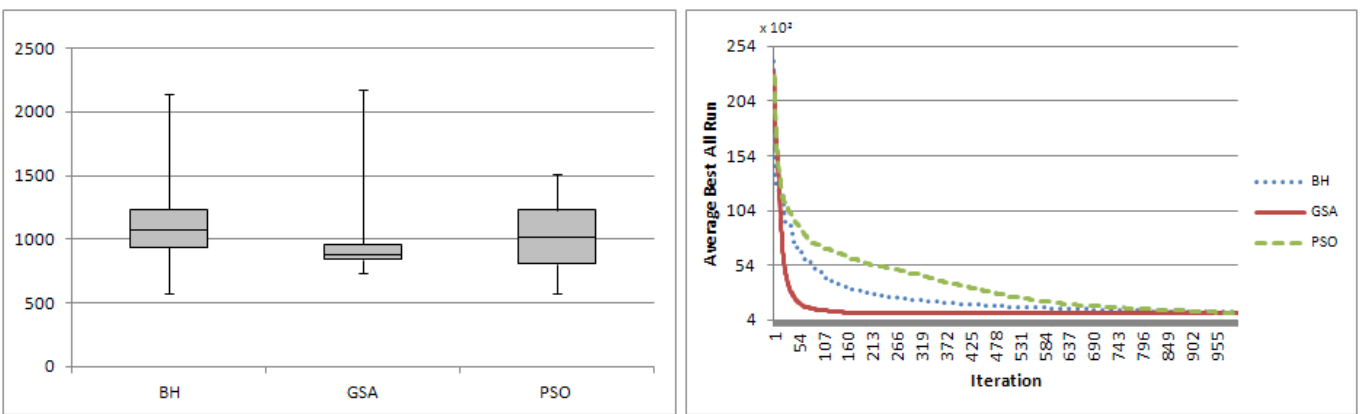

Figure 7 Shifted and rotated Rosenbrock function
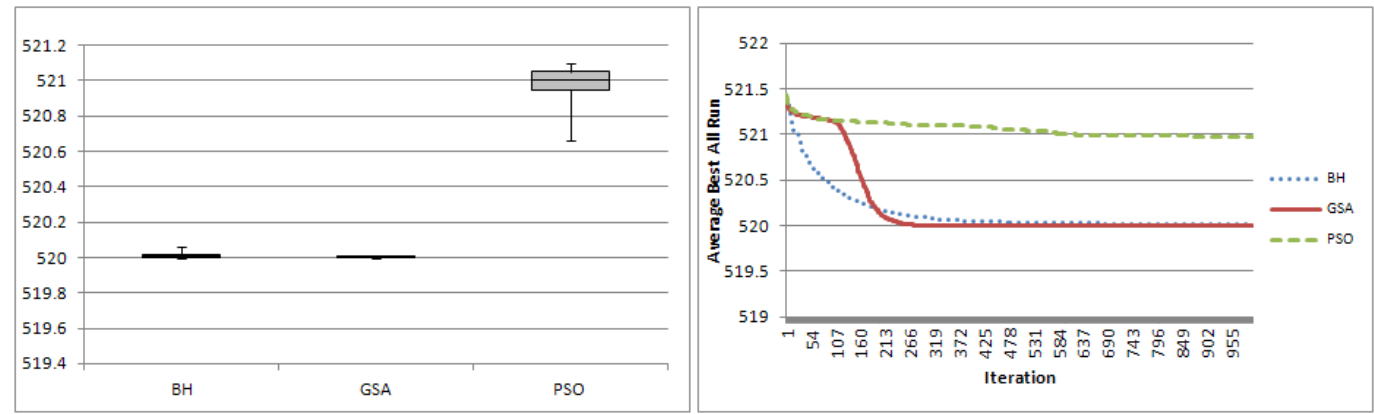

Figure 8 Shifted and rotated Ackley function 

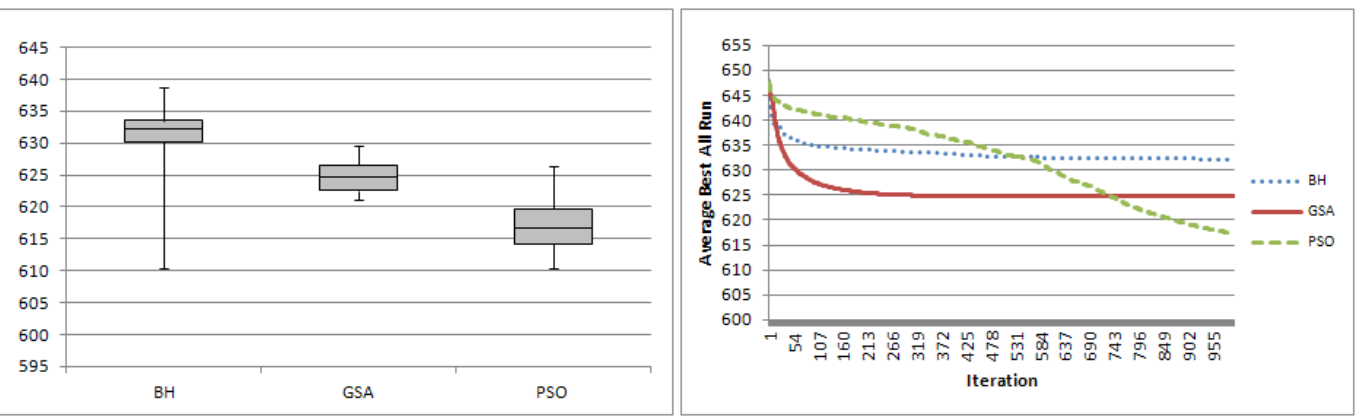

Figure 9 Shifted and rotated Weiestrass function
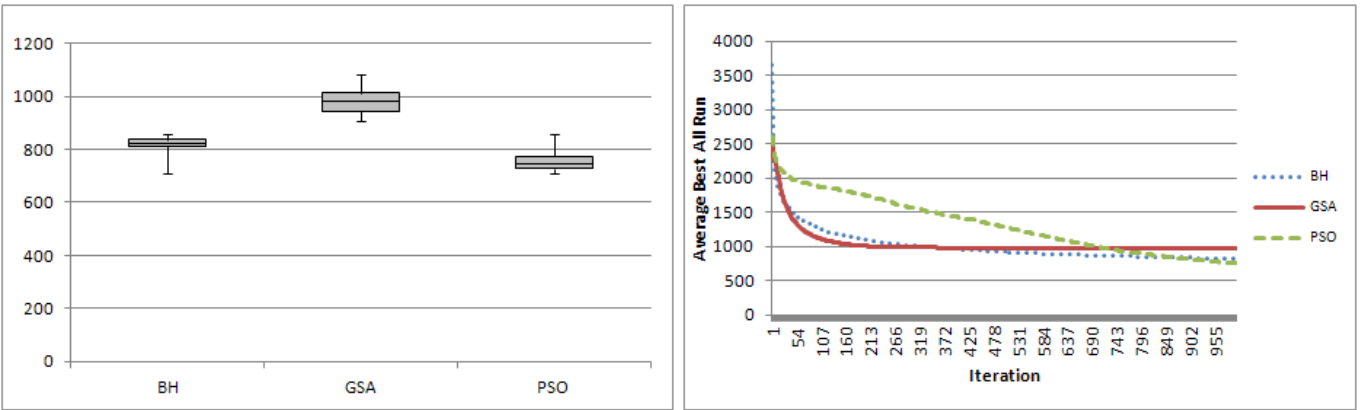

Figure 10 Shifted and rotated Griewank function

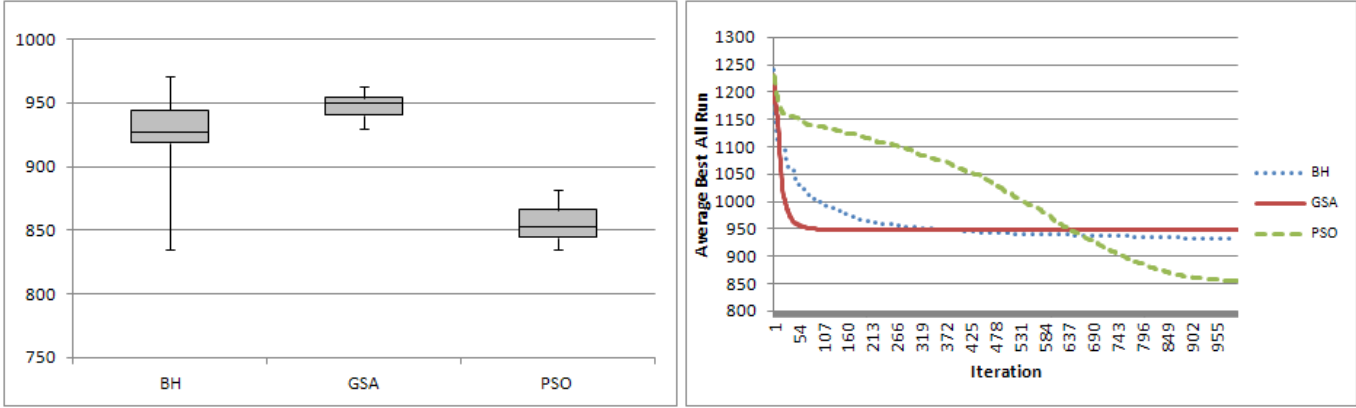

Figure 11 Shifted Rastrigin function
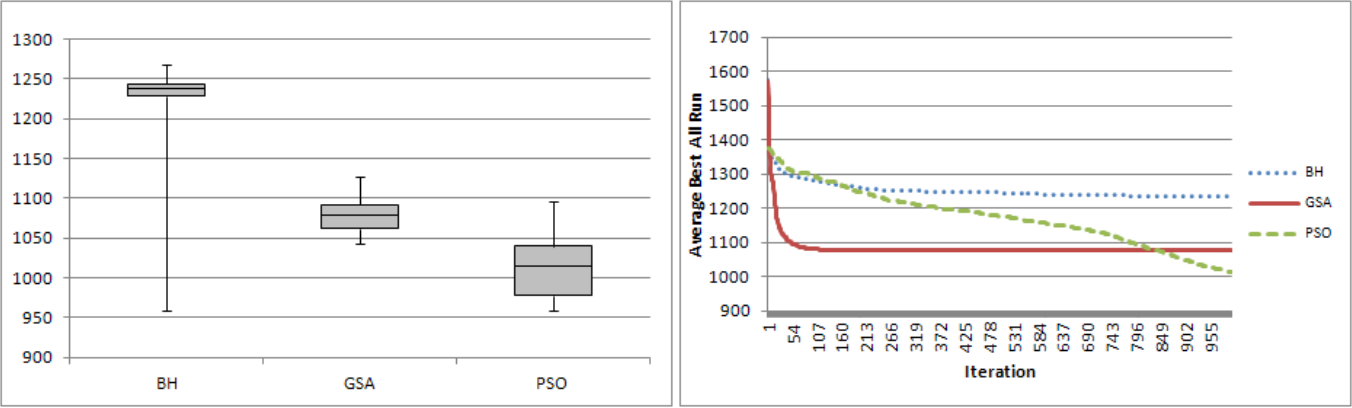

Figure 12 Shifted and rotated Rastrigin function 

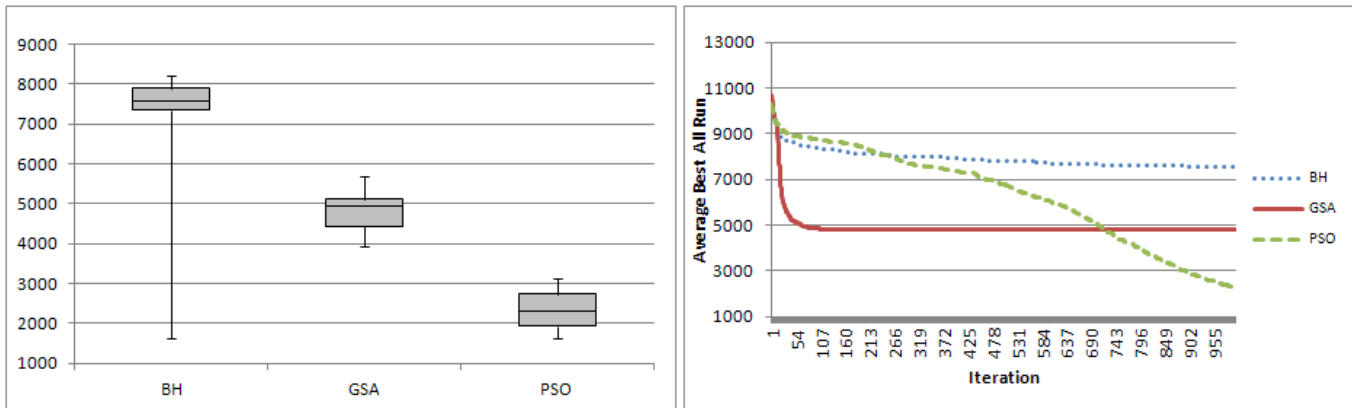

Figure 13 Shifted Schwefel function
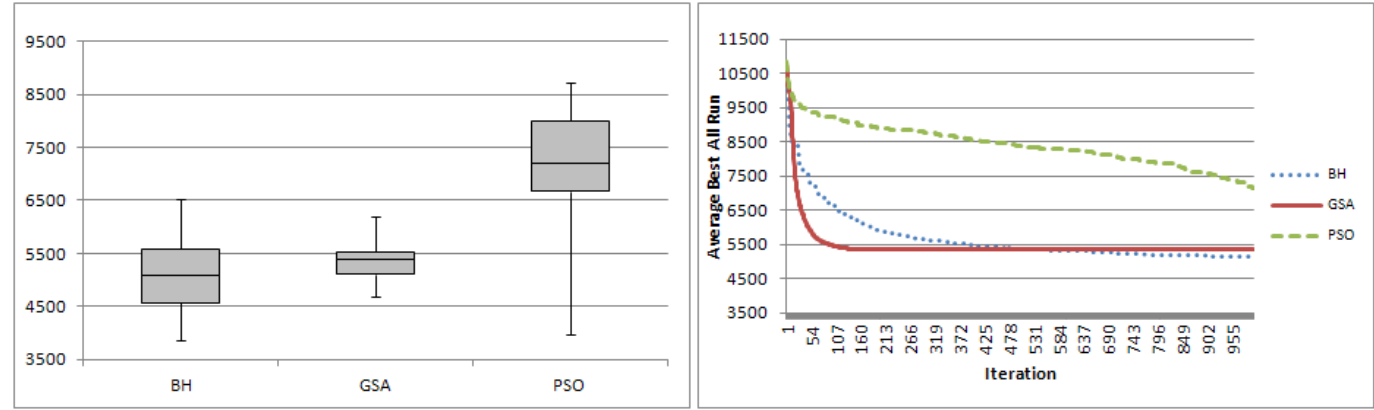

Figure 14 Shifted and rotated Schwefel function
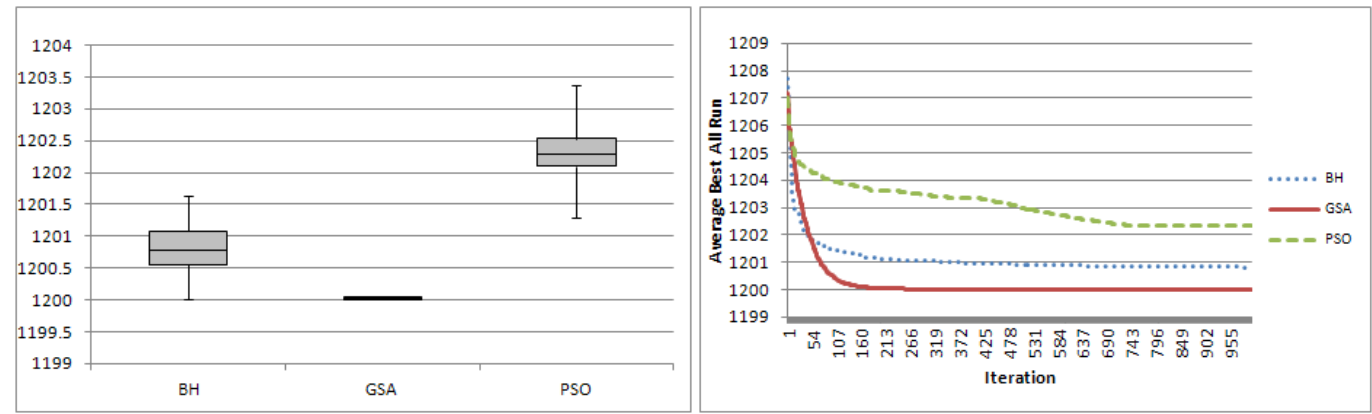

Figure 15 Shifted and rotated Katsuura function

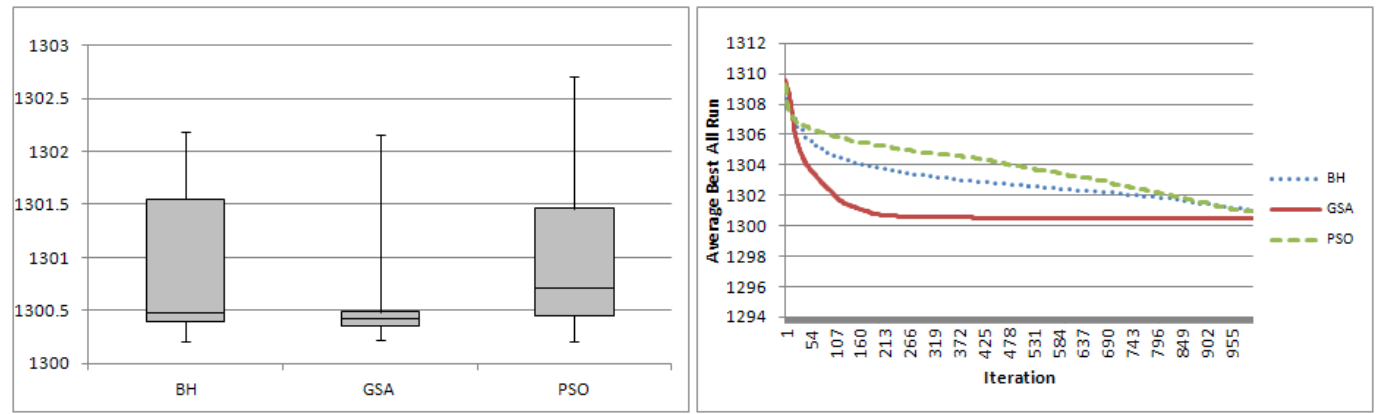

Figure 16 Shifted and rotated HappyCat function 

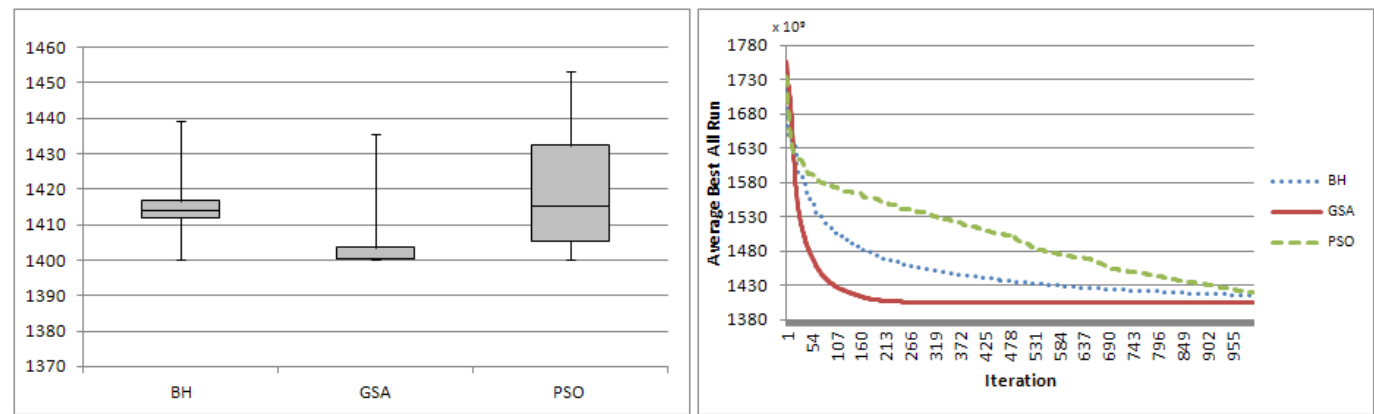

Figure 17 Shifted and rotated HgBat function
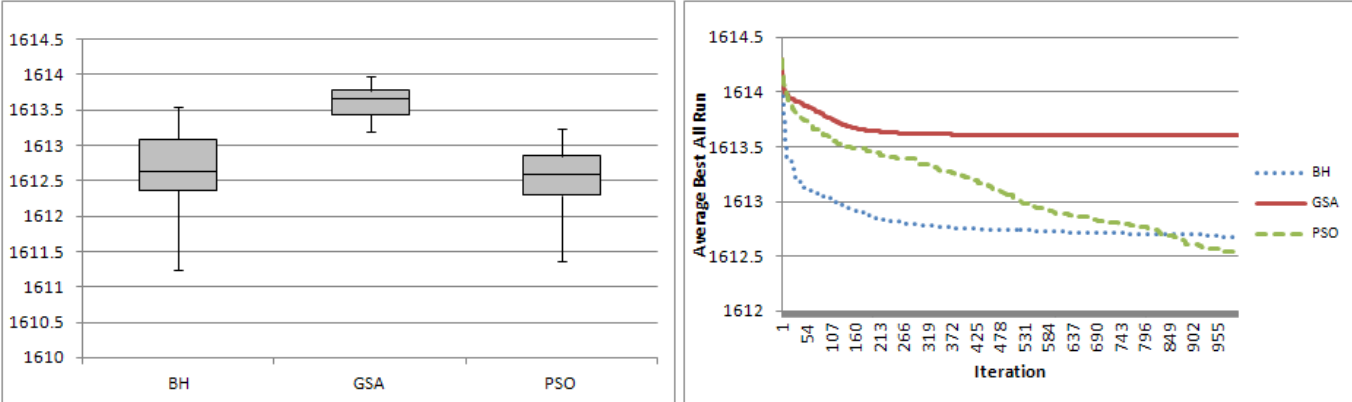

Figure 18 Shifted and rotated Expanded Scaffer F6 function
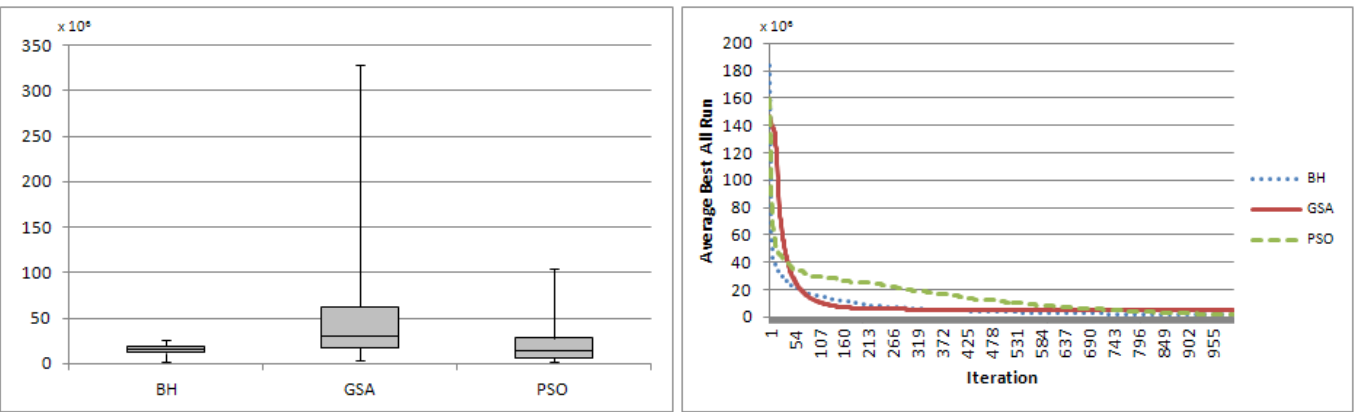

Figure 19 Hybrid Function 1 (N=3)
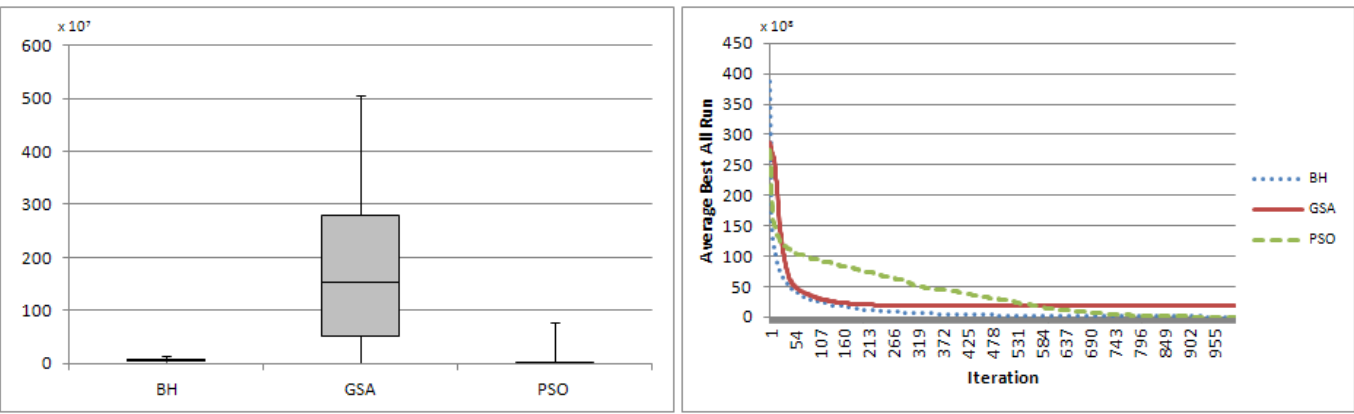

Figure 20 Hybrid Function 2 (N=3) 

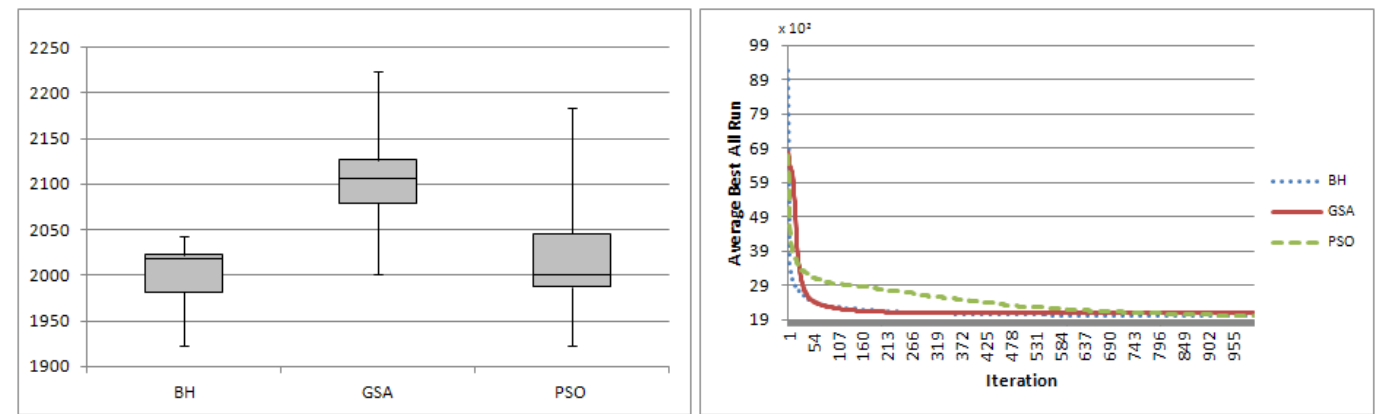

Figure 21 Hybrid Function 3 (N=4)
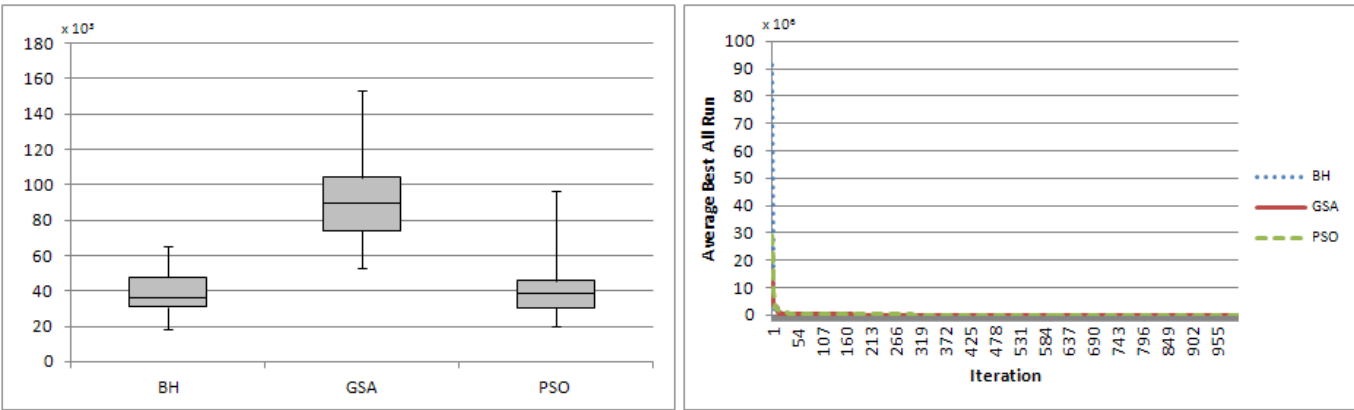

Figure 22 Hybrid Function 4 (N=4)
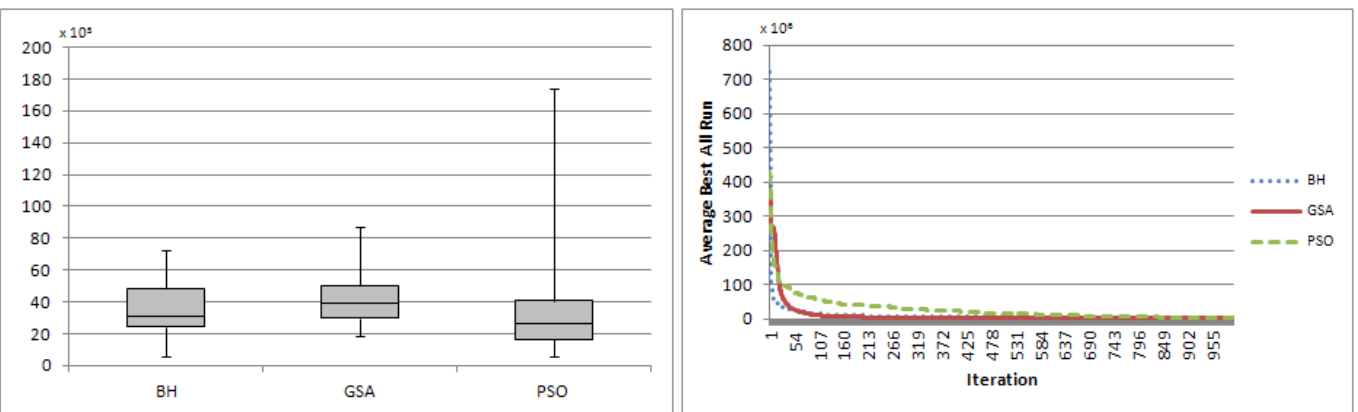

Figure 23 Hybrid Function 5 (N=5)

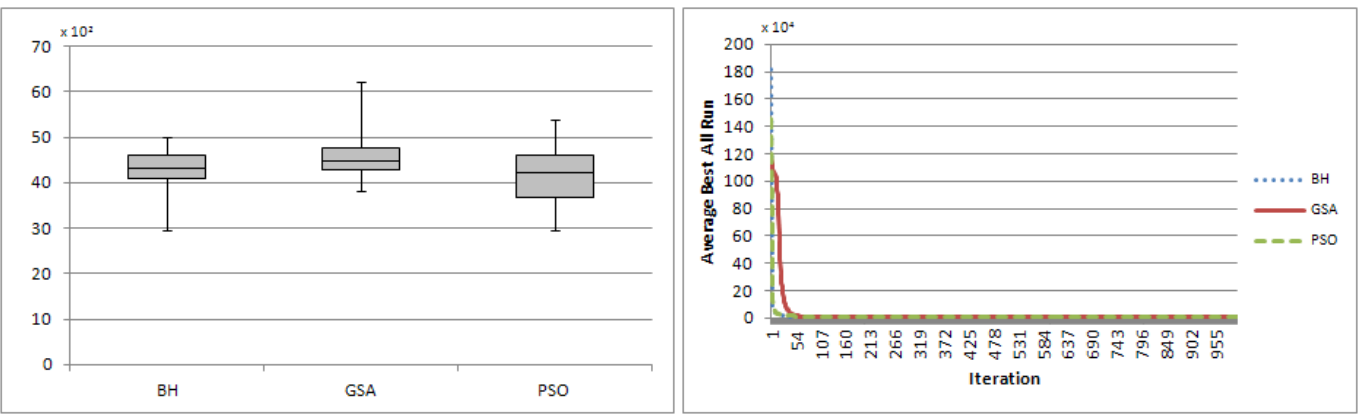

Figure 24 Hybrid Function 6 (N=5) 